\section{EDUCATION}

Research, Imovertion and Solutions on-line $\Theta^{\Theta}$
PSYCHOLOGY

$1+D+i$
Electronic Journal of Research

in Educational Psychology

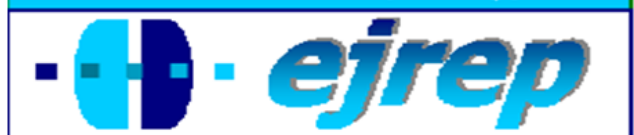

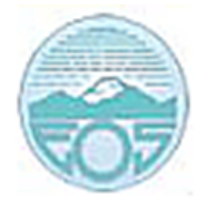

Editorial EOS

\title{
Usos situados de las TIC y mediación de la actividad conjunta en una
} secuencia instruccional de educación primaria ${ }^{1}$

\section{César Coll Salvador, María José Rochera Villach, Rosa Colomina Álvarez}

Dpto. de Psicología Evolutiva y de la Educación, Universidad de Barcelona

\section{España}

Correspondencia: César Coll Salvador. Pg. de la Vall d'Hebrón, 171. 08035 Barcelona (España). E-mail: ccoll@ub.edu

(C) Education \& Psychology I+D+i and Editorial EOS (Spain)

\footnotetext{
${ }^{1}$ Algunos de los planteamientos que se presentan en el texto son tributarios de los resultados teóricos y empíricos obtenidos en el marco del proyecto de investigación "Interacción e influencia educativa: la construcción del conocimiento en entornos electrónicos de enseñanza y aprendizaje", subvencionado por la Comisión Interministerial de Ciencia y Tecnología (proyecto BSO2001-3680-CO2-0; Investigador principal: César Coll).
} 


\section{Resumen}

Introducción. Desde una perspectiva socioconstructivista y situada de los procesos de enseñanza y aprendizaje, en este trabajo se analizan los usos de las tecnologías de la información y la comunicación -TIC- que llevan a cabo una profesora y 19 alumnos de sexto de educación primaria. El estudio se centra en el papel mediador de las TIC en la interacción y en su potencial para innovar los procesos de enseñanza y aprendizaje en el aula.

Método. Utilizando una metodología de estudio de casos, se ha procedido a registrar y analizar una secuencia instruccional sobre la metodología científica en ciencias naturales en la que los alumnos trabajan en pequeños grupos. El análisis de los datos se ha realizado mediante un modelo de análisis de la interacción educativa que permite identificar las formas de organización de la actividad conjunta, y mediante una tipología de usos de las TIC que median en la interactividad.

Resultados. Los resultados muestran usos diversos de las TIC en función de las fases y momentos de la secuencia instruccional y de las características y exigencias que plantean las tareas en cada una de ellas. Asimismo, los resultados ponen de relieve que las TIC se utilizan prioritariamente para mediar las relaciones entre alumnos y contenido y, en menor medida, para mediar las relaciones entre profesora, alumnos y contenidos de aprendizaje.

Discusión y Conclusión. Se concluye que la capacidad de las TIC para transformar las prácticas educativas en el aula depende del uso situado que se hace de ellas en situaciones auténticas de enseñanza y aprendizaje y de las nuevas posibilidades educativas que introducen para mediar la actividad conjunta entre profesor y alumnos.

Palabras Clave: Usos educativos de las TIC. Actividad conjunta. Tareas auténticas. Influencia educativa. Educación primaria.

Recibido: 21/12/09 Aceptación Inicial: 23/12/09 Aceptación Definitiva: 30/04/10 


\title{
Situated uses of ICT and mediation of joint activity in a primary education instructional sequence
}

\begin{abstract}
Introduction. From a socioconstructivist and situated perspective of teaching and learning processes, the authors analyze how one teacher and her group of 19 6th grade pupils use ICT. The study focuses on the way these tools mediate their activity, and evaluates the tools' potential for teaching and learning innovation.

Method. Using a case study method, the development of an instructional unit of natural sciences with pupils working in small groups was analyzed. The sequence was divided into three phases comprising different sessions. A model of analysis of educational interaction was applied, based on: a) the identification and characterization of the forms of joint activity, and b) a typology of uses of ICT focusing on the joint activity of the student, the content and the teacher.
\end{abstract}

Results. Different uses of ICT are identified, linked to the different phases of the instructional sequence and to the features of the tasks and the challenges posed by them in each of these phases. Furthermore, the results show that the ICT are primarily used to mediate between pupils and content but, to a lesser extent, between pupils, the content, and the teacher.

Discussion and Conclusion. In conclusion the capacity of ICT's to transform educational practice in the classroom depends on the participant's situated use of these tools in authentic teaching and learning situations, and also on the new affordances that these tools introduce to the mediation of the participants' joint activity.

Keywords: Educational uses of ICT, Joint activity, Authentic tasks, Educational influence, Primary education.

Received: 12/21/09 Initial Acceptance: 12/23/09 Definitive Acceptance: 04/30/10 


\section{Introducción}

El uso de las tecnologías de la información y la comunicación - TIC - en las aulas para promover el aprendizaje se ha extendido considerablemente en el transcurso de las últimas décadas. El aprendizaje del funcionamiento de los ordenadores -hardware-, el uso de programas informáticos -software- y la utilización de Internet para buscar, representar y comunicar información se han ido incorporando progresivamente a la educación primaria dando lugar a un amplio abanico de situaciones que abarca desde usos muy puntuales hasta la configuración de entornos electrónicos de aprendizaje. Sin embargo, las investigaciones realizadas hasta el momento muestran que la incorporación de las TIC a la educación en general, y a la educación primaria en particular, no ha dado lugar a la mejora esperada en los aprendizajes de los alumnos y que el uso que se hace de estas tecnologías es en general muy restrictivo (ver, por ejemplo, Barquín, 2007; Cuban, 2001; Zhao y Frank, 2003).

Así, de los tres tipos de usos básicos de las TIC identificados por Muraro (2005), a saber, las TIC como objeto de aprendizaje -relativo al uso de la tecnología como conjunto de aprendizajes que se incluyen como materia en un currículo-, las TIC como instrumento para aprender, -relativo al uso de la tecnología como recurso para resolver problemas en relación con otros contenidos curriculares- y las TIC como instrumento para enseñar -relativo al uso de la tecnología como recurso didáctico del profesor-, los estudios coinciden en señalar que en la educación primaria el primero es, con diferencia, el más frecuente.

Entre los factores que explican esta infrautilización de las TIC y el escaso aprovechamiento de las posibilidades que ofrecen para innovar y mejorar las prácticas educativas cabe señalar la falta de equipamiento en las aulas, la escasez de software educativo, la deficiente difusión y el escaso conocimiento del software existente y sobre todo una formación docente aún deficitaria en los usos educativos de las TIC (Colás y Jiménez, 2008). Algunas investigaciones muestran que los profesores tienden a incorporar las tecnologías en las situaciones en las que no se requieren grandes cambios en relación con la docencia que ya realizan sin el uso de las TIC (Zhao y Frank, 2003) y que el principal obstáculo para la innovación educativa apoyada en las TIC se encuentra a menudo en la propias prácticas docentes del profesorado (Sigalés, Mominó y Meneses, 2009). 
Así, por una parte, las características organizativas y estructurales de los centros escolares conforman un marco institucional para la incorporación de las TIC que favorece y potencia unos usos educativos determinados de estas tecnologías. Por otra parte, la manera como el profesorado utiliza las TIC depende de los marcos teóricos que adopta y de sus creencias sobre la utilidad educativa de estas tecnologías. En lo que concierne al primer aspecto, la flexibilización de espacios y tiempos facilita la aparición de usos innovadores de la tecnología permitiendo diversificar los contextos de realización de experiencias educativas y variar la temporalización para llevarlas a cabo (Fernández y Correa, 2009). En relación al segundo aspecto, algunos estudios muestran que el hecho de que el profesorado tenga una perspectiva constructivista de los procesos de enseñanza y aprendizaje es un predictor de los usos de las TIC que el mismo profesorado considera positivos para el aprendizaje (Becker, 2000; Blantoon, Moorman y Trathen, 1998; Tondeur, Van Braak y Valcke, 2007). Y los estudios sobre las creencias de los docentes sobre las TIC muestran que, a pesar de que éstos las valoran muy positivamente para mejorar la participación de los alumnos y la colaboración en grupo, para estimular su interés y para facilitar un aprendizaje autónomo, en realidad las utilizan en el aula con estas finalidades muy escasamente (Aera, 2005; Instituto Nacional de Evaluación y Asesoramiento Educativo, Neturity y Fundación Germán Sánchez Ruipérez, 2007).

En definitiva, a la luz de los resultados de los estudios anteriores se plantea el desafío de investigar sobre los usos y los contextos de uso que permiten innovar y mejorar las prácticas educativas con el apoyo de las TIC para lograr los objetivos básicos de la educación aprender a conocer, aprender a hacer y aprender a convivir y a ser-, y dar respuestas a las nuevas demandas que la sociedad de la información exige (Coll y Rodríguez Illera, 2008; Marchesi y Díaz, 2009). Un aspecto clave de esta indagación consiste en analizar si la incorporación de las TIC a las aulas explota realmente las posibilidades que estas tecnologías ofrecen para resolver situaciones y problemas relevantes para los alumnos en la sociedad actual, lo que significa dirigir la atención no sólo a los usos efectivos que se hacen de las TIC sino también al nivel de autenticidad, de contextualización y de relevancia que comportan estos usos.

En este marco, el presente trabajo tiene un doble objetivo. En primer lugar, describir y analizar los usos reales de las TIC que realizan una profesora y sus alumnos de educación primaria en un entorno presencial de enseñanza y aprendizaje mientras llevan a cabo una actividad que podemos calificar como auténtica. En segundo lugar, y en estrecha relación con el 
objetivo anterior, el estudio pretende proporcionar algunos criterios que permiten caracterizar el papel más o menos transformador e innovador de los usos de las TIC identificados.

\section{El uso educativo de las TIC en el aula desde una perspectiva socioconstructivista}

La perspectiva constructivista, comunicativa y cultural de los procesos de enseñanza y aprendizaje en la que se fundamenta este trabajo postula que el aprendizaje es un proceso social y situado (Palincsar, 1998; Wenger, 1988). Desde esta perspectiva, se subraya que el aprendizaje los alumnos llevan a cabo tiene lugar en su actividad conjunta con el profesor en torno a los contenidos y tareas escolares, al tiempo que se destaca la función mediadora del profesor como guía y orientador de estos procesos (Coll, 2001). La incorporación de las TIC en el aula con estos postulados constructivistas plantea un doble reto: el de comprender cómo su uso puede mediar las formas de organización de la actividad conjunta de profesor y alumnos a lo largo de un proceso de enseñanza y aprendizaje, y el de estudiar cómo el profesorado ayuda a los alumnos y ajusta sus ayudas en el transcurso de esta actividad, gracias a las tecnologías (Coll, Mauri y Onrubia, 2008; Coll, Onrubia y Mauri, 2007).

Así mismo, el enfoque constructivista de orientación social, cultural y situada pone de relieve algunas dimensiones fundamentales de los procesos de enseñanza y aprendizaje cuando son estudiados desde la perspectiva de la autenticidad. Por una parte, las tareas de aprendizaje en torno a las cuales se organiza la actividad conjunta deben respetar en lo posible las mismas condiciones y plantear al alumnado exigencias similares en lo que concierne al uso de las TIC que las que se requieren en situaciones reales. Estos requerimientos tienen como finalidad provocar desafíos intelectuales complejos y aprendizajes con un elevado grado de significatividad. Por otra parte, debe promoverse un uso autónomo y situado de la tecnología que ayude a los alumnos a utilizar estratégicamente su conocimiento sobre las TIC en función de las condiciones, características y demandas del contexto y de la tarea (Coll, Rochera y Onrubia, 2009; Darling-Hammond, Ancess y Falk, 1995; Díaz Barriga, 2005). 
Tabla 1. La función mediadora de las tecnologías de la información y de la comunicaciónTIC- en las relaciones entre los elementos del triángulo interactivo (adaptado de Coll, 2004; Coll, Mauri y Onrubia, 2008).

\begin{tabular}{|c|c|c|}
\hline Uso de las TIC & Función mediadora & Caracterización y ejemplos \\
\hline
\end{tabular}

Las TIC como
objeto de aprendiza-
je

Las TIC como instrumentos mediadores de las relaciones entre los alumnos y los contenidos (y tareas) de aprendizaje

Las TIC como instrumentos mediadores de las relaciones entre los profesores y los contenidos

Las TIC como instrumentos mediadores de las relaciones entre los profesores y los alumnos o entre los alumnos

Las TIC como instrumentos mediadores de la actividad conjunta desplegada por profesores $y$ alumnos durante la realización de las tareas o actividades de enseñanza aprendizaje

Las TIC como instrumentos configuradores de entornos o espacios de trabajo y de aprendizaje
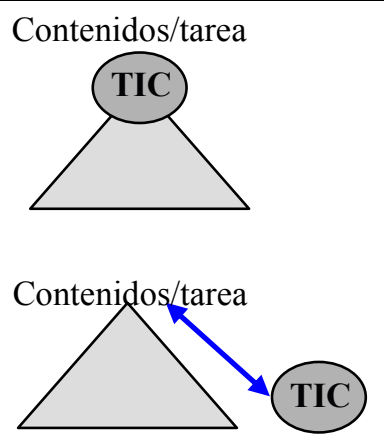

Alumnos

\section{Contenidos/tarea}

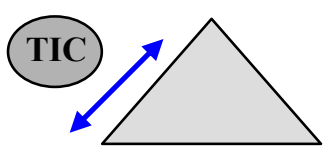

Profesor

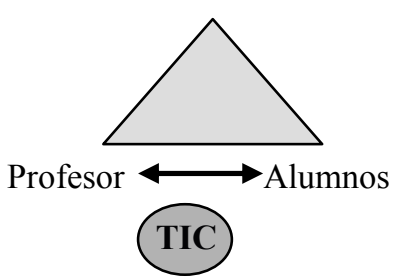

Contenidos/tarea
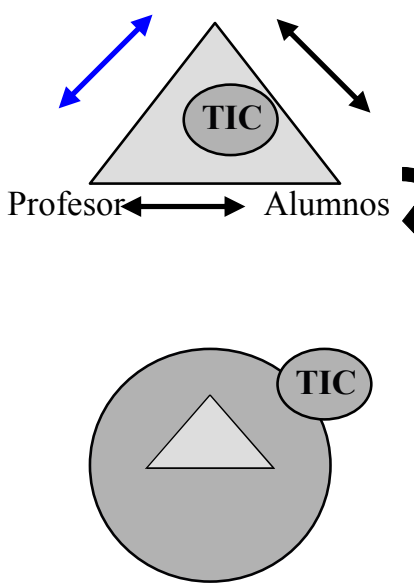

Buscar y seleccionar contenidos de aprendizaje; acceder a repositorios de contenidos y o tareas de aprendizaje; realizar tareas y actividades de aprendizaje

Aprender /enseñar contenidos, el funcionamiento de los ordenadores, sus utilidades y aplicaciones, características de la utilización de internet, el manejo de redes de trabajo con ordenadores, etc.

Acceder a repositorios de objetos de aprendizaje y/o a bases de datos y bancos de propuestas de actividades; elaborar y mantener registros de las actividades de enseñanza y aprendizaje realizadas; planificar y preparar actividades

Llevar a cabo intercambios comunicativos entre profesores y alumnos o entre los estudiantes no directamente relacionados con los contenidos o las tareas (presentación personal, solicitud de información personal o general, saludos, expresión de sentimientos, etc.)

Ayudar a amplificar determinadas actuaciones del profesor (explicar, ilustrar, relacionar, etc. mediante el uso de presentaciones, simulaciones, modelizaciones, etc.); ayudar a amplificar determinadas actuaciones de los alumnos (hacer aportaciones, intercambiar informaciones y propuestas, mostrar los avances y los resultados); realizar un seguimiento de los avances y dificultades de los alumnos.

Configurar entornos o espacios de aprendizaje individual en línea (por ejemplo, materiales destinados al aprendizaje autónomo); configurar entornos o espacios de trabajo colaborativo en línea (por ejemplo, las herramientas y los entornos CSCL -Computer-Supported Collaborative Learning); configurar entornos o espacios de actividad en línea que se desarrollan en paralelo. 
Para estudiar cómo se utilizan las TIC en entornos de enseñanza y aprendizaje y su relación con el tipo de mediación en la actividad conjunta que promueven, se parte de una tipología de usos conceptualmente fundamentada en la perspectiva constructivista de orientación sociocultural (Coll, 2004). Esta categorización se apoya en la idea de que la diferencia esencial entre los usos de las TIC en lo que concierne a su capacidad para transformar las prácticas educativas en el aula no depende tanto de las características intrínsecas de los recursos tecnológicos como de su uso en el espacio delimitado por las relaciones entre los tres elementos del triángulo interactivo: alumnos, profesor y contenido o tarea de aprendizaje (Coll, Onrubia y Mauri, 2008). La Tabla 1 sintetiza esta propuesta de usos.

Como se señalaba anteriormente, esta categorización permite dar cuenta del papel mediador de las TIC en la actividad conjunta y comprender su potencialidad educativa, puesto que, desde esta perspectiva teórica, es en el marco de la interacción que se establece entre profesor y alumnos mientras abordan los contenidos y las tareas de aprendizaje donde es posible ajustar la ayuda educativa. Así, la incorporación de las tecnologías al aula no conduce automática ni linealmente a la transformación de los procesos de enseñanza y aprendizaje. En este planteamiento la clave está en identificar los usos que profesores y alumnos hacen de las TIC mientras despliegan su actividad en el aula, y en analizar los cambios que estas tecnologías introducen, a través de esos usos, en la organización de la actividad conjunta mediando las relaciones entre alumnos, profesor y contenidos o tareas de aprendizaje.

\section{Método}

\section{Participantes}

El caso estudiado es una secuencia instruccional sobre ciencias de la naturaleza en la que participaron una profesora y 19 estudiantes de sexto curso de educación primaria (12 años), que ya contaban con experiencias previas de trabajo conjunto en actividades y tareas de características similares. La profesora tenía una amplia práctica docente en el diseño y desarrollo de entornos de enseñanza y aprendizaje que incorporan las $\mathrm{TIC}^{2}$.

\footnotetext{
${ }^{2}$ Quisiéramos agradecer a la profesora y al alumnado que ha participado en esta investigación su colaboración y disposición durante todo el desarrollo de la misma.
} 


\section{Instrumentos}

Se han utilizado distintos instrumentos y procedimientos para la recogida de información de la secuencia instruccional objeto de estudio: registro video, audio, narrativo y electrónico, que constituyen el corpus principal de datos. Adicionalmente, se han realizado entrevistas a la profesora y a una muestra de alumnos, antes y después de la secuencia, se ha recabado documentación relativa a la planificación, y se han recogido los documentos generados por los participantes en el trancurso de la misma.

El análisis de los datos se ha llevado a cabo mediante instrumentos de análisis diferenciados para identificar los usos de las TIC, y para identificar las formas de organización de la actividad conjunta en la que esos usos se incardinan. Para la identificación y análisis de los usos de las TIC se ha utilizado una tipología de usos elaborada desde una perspectiva constructivista (Coll, 2004) que contempla las siguientes categorías: a) uso de las TIC como contenidos de aprendizaje, b) uso de las TIC como repositorios de contenidos de aprendizaje, c) uso de las TIC como herramientas de búsqueda y selección de contenidos de aprendizaje, d) uso de las TIC como instrumentos cognitivos a disposición de los participantes, d) uso de las TIC como auxiliares o amplificadores de la actuación docente, y e) uso de las TIC como herramientas de comunicación entre los participantes. Para la identificación y análisis de las formas de organización de la actividad conjunta y su evolución en el transcurso del proceso instruccional se ha utilizado el modelo de análisis de la interactividad (Coll, Colomina, Onrubia y Rochera, 1992; Coll, Onrubia y Mauri, 2008; Colomina, Onrubia y Rochera, 2001). El modelo permite identificar en cada una de las sesiones de la secuencia instruccional diferentes segmentos de interactividad -0 formas de organización de la actividad conjunta-, a partir de la estructura de participación que regula las actuaciones de los participantes, es decir, quién puede decir o hacer qué, con quién, cómo, cuándo, para obtener qué resultado y utilizando qué recursos.

\section{Procedimiento}

De acuerdo con una metodología de análisis cualitativo de casos (Stake, 1994; Yin, 2006), se ha llevado a cabo el estudio observacional de la secuencia instruccional en su totalidad. En esta secuencia se trataba de desarrollar un proyecto para la educación sobre el clima y la meteorología -el proyecto Edumet- durante un total de 20 sesiones desplegadas a lo largo 
del segundo y tercer trimestre del curso escolar. La secuencia se organizó en torno a actividades de enseñanza y aprendizaje en modalidad presencial que trataban de replicar los pasos de la investigación científica: planteamiento de alguna pregunta sobre el clima y la meteorología, recogida de datos meteorológicos, análisis de resultados y establecimiento de conclusiones. La realización de las actividades se apoyaba en el trabajo en pequeño grupo y el uso de las TIC en el aula. Se utilizaban, además, recursos telemáticos comunicativos y colaborativos (correo electrónico, foros) para compartir datos y conclusiones con diferentes escuelas que participaban en el proyecto. El proyecto estaba organizado en tres fases dirigidas respectivamente a la presentación del mismo y de las escuelas participantes - 6 sesiones-, a la construcción de una base de datos meteorológicos -6 sesiones- y al análisis de esos datos e intercambio de conclusiones -8 sesiones-.

En cada una de las fases, la profesora y los alumnos utilizaron diferentes herramientas tecnológicas para la elaboración de los productos requeridos. Así, en la primera fase la profesora utilizó la página Web del proyecto Edumet para explicar el proyecto, mientras que los alumnos utilizaron un procesador de textos para la construcción de un documento de presentación de la escuela. En la segunda fase los alumnos utilizaron una hoja de cálculo para la construcción de una base de datos meteorológicos. En la tercera fase utilizaron un programa de dibujo para la construcción de la portada final de la investigación, el procesador de textos para la escritura de la investigación y la hoja de cálculo para la elaboración de las gráficas que acompañaban la presentación de los resultados; además, utilizaron el correo electrónico y el foro para intercambiar información entre las escuelas.

El procedimiento de registro de datos consistió en el registro en video de las 20 sesiones de la secuencia en las que, asimismo, se llevaron a cabo registros audio y narrativo, con la participación sistemática de dos investigadores. Además, se registraron electrónicamente los intercambios en línea entre la profesora y los participantes de las otras escuelas. Se llevaron a cabo entrevistas iniciales y finales a la profesora y a una muestra de alumnos (un pequeño grupo de 5 alumnos). Finalmente se procedió a recoger la documentación relativa a la planificación y diseño de la secuencia instruccional, y la documentación elaborada por los participantes durante todo el proceso. 
Análisis de los datos

La identificación y análisis de los usos de las TIC en la secuencia didáctica estudiada se ha llevado a cabo mediante la aplicación de la tipología de usos anteriormente citada. En cada una de las fases del proyecto se ha procedido a identificar los distintos usos predominantes de las TIC de profesora y alumnos, caracterizar tales usos, situarlos en el marco más amplio de la actividad en la que están inscritos y, por último, explicitar la mediación que permiten en la actividad conjunta.

La identificación y análisis de las formas de organización de la actividad conjunta y su evolución en el transcurso del proceso instruccional se ha llevado a cabo siguiendo el modelo de análisis de la interactividad, antes mencionado. De acuerdo con este modelo, en cada una de las sesiones que configuran las tres fases del proyecto, se ha procedido a identificar los segmentos de actividad conjunta según las actuaciones predominantes de los participantes. Además, puesto que la situación estudiada es de carácter presencial con el apoyo de las TIC, se explicita la información relativa a los segmentos que se desarrollan con el uso de las TIC o sin el mismo. Finalmente, la estructura global de la actividad conjunta se representa gráficamente mediante un mapa que recoge la totalidad de segmentos con TIC y sin TIC a lo largo de la secuencia didáctica

La aplicación de ambos tipos de análisis a un mismo corpus de datos permite comprender el uso efectivo que hacen los participantes de los recursos tecnológicos en el marco de la actividad conjunta que desarrollan. Estos análisis se complementan con el del contenido de las entrevistas, siendo esta información utilizada para apoyar la interpretación de los resultados obtenidos del análisis de las informaciones sobre el desarrollo de la secuencia de enseñanza y aprendizaje.

\section{Resultados}

Los usos de las TIC en la secuencia instruccional

En las tres fases del desarrollo del proyecto Edumet se han podido identificar diferentes usos por parte de la profesora y de los alumnos, que se describen en la Tabla 2. En la pri- 
mera columna de la tabla se indican los tipos de uso de las TIC, en la segunda columna aparecen los porcentajes de aparición de los distintos tipos de uso, y en la tercera se describen brevemente. Las dos últimas columnas sitúan los distintos usos en el marco de la actividad conjunta de la profesora y los estudiantes, e identifican el tipo de mediación que comportan.

Tabla 2. Usos y mediación de las TIC en las tres fases de la secuencia instruccional

\begin{tabular}{ccccc}
\hline $\begin{array}{c}\text { Tipos de usos de } \\
\text { TIC }\end{array}$ & $\%$ & $\begin{array}{c}\text { Descripción del uso } \\
\text { de TIC }\end{array}$ & $\begin{array}{c}\text { Actividad conjunta } \\
\text { en la que se inserta } \\
\text { las TIC }\end{array}$ & $\begin{array}{c}\text { Uso de las TIC para } \\
\text { mediar como... }\end{array}$ \\
& & &
\end{tabular}

FASE 1. Presentación del proyecto Edumet. Presentación de las escuelas participantes

(\% uso TIC en la fase: $27,29 \%$ )

\begin{tabular}{lll}
\hline TIC para definir el & $1,08 \%$ & La profesora ofrece \\
espacio/procedi- & & información sobre el \\
miento de realiza- & & uso de los espacios de \\
ción de la tarea & & la Web Edumet: co- \\
(Profesora) & & municación y base de \\
& & datos
\end{tabular}

TIC como instrumento de amplificación de la acción docente (Profesora)
Actividad conjunta entre profesora $y$ alumnos en torno a la presentación del proyecto Edumet
Instrumentos mediadores de la actividad conjunta desplegada por profesores $y$ alumnos durante la realización de las tareas de enseñanza/aprendizaje proyecto Edumet
Las TIC como

objeto de aprendizaje

\begin{tabular}{ll}
\hline TIC como contenido & $2,41 \%$ \\
de aprendizaje (Pro- & La profesora explica la \\
fesora) & estructura de ventanas \\
& y cómo salvar un \\
documento sin borrar \\
otro previo
\end{tabular}

TIC como instru- $\quad 0,5 \%$ mento de comunicación (Profesora)
La profesora publica la presentación de la escuela y los mensajes de los alumnos en el foro de la Web Edumet
Actividad conjunta entre profesora y otros participantes de otras escuelas
Las TIC como instrumentos mediadores de las relaciones entre los profesores y los alumnos o entre los alumnos

\begin{tabular}{|c|c|c|c|c|}
\hline Total Profesora & $11,84 \%$ & & & \\
\hline $\begin{array}{l}\text { TIC como instru- } \\
\text { mento cognitivo } \\
(\text { Alumno-Alumno) }\end{array}$ & $83,86 \%$ & $\begin{array}{l}\text { Los alumnos elaboran } \\
\text { un texto de presenta- } \\
\text { ción y mensajes } \\
\text { (Word) para contestar } \\
\text { a otras escuelas }\end{array}$ & \multirow[t]{2}{*}{$\begin{array}{l}\text { Actividad conjunta } \\
\text { entre alumnos (peque- } \\
\text { ño grupo) en torno a la } \\
\text { elaboración de datos } \\
\text { meteorológicos }\end{array}$} & \multirow[t]{2}{*}{$\begin{array}{l}\text { Las TIC como instru- } \\
\text { mentos mediadores de } \\
\text { las relaciones entre } \\
\text { los alumnos y los } \\
\text { contenidos (y tareas) } \\
\text { de aprendizaje }\end{array}$} \\
\hline $\begin{array}{l}\text { TIC con uso instru- } \\
\text { mental (Alumno- } \\
\text { Alumno) }\end{array}$ & $4,30 \%$ & $\begin{array}{l}\text { Los alumnos formate- } \\
\text { an, guardan, copian, } \\
\text { imprimen los docu- } \\
\text { mentos }\end{array}$ & & \\
\hline
\end{tabular}


FASE 2. Construcción de una base de datos meteorológicos (\% uso TIC en la fase: $4,83 \%$ )

\begin{tabular}{lllll}
\hline $\begin{array}{l}\text { TIC con uso instru- } \\
\text { mental (Alumno- }\end{array}$ & $100 \%$ & $\begin{array}{l}\text { Los estudiantes copian } \\
\text { los datos del papel a la } \\
\text { Alumno) }\end{array}$ & $\begin{array}{l}\text { Actividad conjunta } \\
\text { entre alumnos en torno datos } \\
\text { a la recogida de datos } \\
\text { en grupo }\end{array}$ & $\begin{array}{l}\text { Las TIC como instru- } \\
\text { las relaciones entre } \\
\text { los alumnos y los } \\
\text { contenidos (y tareas) } \\
\text { de aprendizaje }\end{array}$ \\
\hline Total alumnos & $100 \%$ & & & \\
\hline
\end{tabular}

FASE 3. Elaboración de una investigación a partir de la base de datos e intercambio de

\section{conclusiones}

(\% uso TIC en la fase: $80,53 \%)$

\begin{tabular}{|c|c|c|c|c|}
\hline $\begin{array}{l}\text { TIC con uso instru- } \\
\text { mental } \\
\text { (Alumno-Alumno) }\end{array}$ & $76,60 \%$ & $\begin{array}{l}\text { Los alumnos formate- } \\
\text { an, guardan, copian, } \\
\text { imprimen, los docu- } \\
\text { mentos } \\
\text { Los alumnos copian } \\
\text { un texto de presenta- } \\
\text { ción que han elabora- } \\
\text { do previamente en } \\
\text { papel }\end{array}$ & \multirow[t]{2}{*}{$\begin{array}{l}\text { Actividad conjunta } \\
\text { entre alumnos en } \\
\text { torno a la elaboración } \\
\text { de datos en pequeño } \\
\text { grupo }\end{array}$} & \multirow[t]{2}{*}{$\begin{array}{l}\text { Las TIC como instru- } \\
\text { mentos mediadores de } \\
\text { las relaciones entre los } \\
\text { alumnos y los conteni- } \\
\text { dos (y tareas) de } \\
\text { aprendizaje }\end{array}$} \\
\hline $\begin{array}{l}\text { TIC como instru- } \\
\text { mento cognitivo } \\
\text { (Alumno-Alumno) }\end{array}$ & $14,11 \%$ & $\begin{array}{l}\text { Los alumnos elaboran } \\
\text { mensajes (Word) para } \\
\text { contestar a alumnos de } \\
\text { otras escuelas y un } \\
\text { gráfico excel con } \\
\text { datos meteorológicos }\end{array}$ & & \\
\hline Total alumnos & $91,71 \%$ & & & \\
\hline $\begin{array}{l}\text { TIC como contenido } \\
\text { de aprendizaje (Pro- } \\
\text { fesora) }\end{array}$ & $7,33 \%$ & $\begin{array}{l}\text { La profesora explica el } \\
\text { programa Print Artist }\end{array}$ & $\begin{array}{l}\text { Actividad conjunta } \\
\text { entre profesora y } \\
\text { alumnos en torno a la } \\
\text { elaboración del pro- } \\
\text { yecto }\end{array}$ & $\begin{array}{l}\text { Las TIC como } \\
\text { objeto de aprendizaje }\end{array}$ \\
\hline $\begin{array}{l}\text { TIC como instru- } \\
\text { mento de comunica- } \\
\text { ción (Profesora) }\end{array}$ & $0,96 \%$ & $\begin{array}{l}\text { La profesora publica } \\
\text { la presentación de la } \\
\text { escuela en la Web y } \\
\text { los mensajes en el foro } \\
\text { Edumet }\end{array}$ & $\begin{array}{l}\text { Actividad conjunta } \\
\text { entre profesora y otros } \\
\text { participantes de otras } \\
\text { escuelas }\end{array}$ & $\begin{array}{l}\text { Llevar a cabo inter- } \\
\text { cambios comunicati- } \\
\text { vos entre profesores y } \\
\text { alumnos o entre los } \\
\text { estudiantes no direc- } \\
\text { tamente relacionados } \\
\text { con los contenidos o } \\
\text { las tareas (presentación } \\
\text { personal, solicitud de } \\
\text { información personal o } \\
\text { general, saludos, ex- } \\
\text { presión de sentimien- } \\
\text { tos, etc.) }\end{array}$ \\
\hline Total profesora & $8,29 \%$ & & & \\
\hline
\end{tabular}

Durante la fase 1 -presentación del proyecto Edumet, presentación de las escuelas- las TIC son utilizadas durante el 27,29 \% del tiempo total de duración de la fase. Del tiempo total de uso de las tecnologías, un $11,84 \%$ corresponde a la profesora y un $88,16 \%$ a los alumnos. 
La profesora usa las TIC como apoyo a la aportación de la información, como contenido de aprendizaje y para definir el espacio y procedimiento de realización de la tarea. Por su parte, los alumnos utilizan las tecnologías fundamentalmente como instrumento cognitivo para elaborar un texto de presentación de su escuela en word y en menor medida hacen un uso instrumental de ellas, consistente en copiar en word un documento en papel. En la fase 2 las TIC se utilizan únicamente un $4,83 \%$ del tiempo total y siempre por parte de los alumnos; se trata siempre de un uso puramente instrumental que consiste en pasar los datos meteorológicos de un plantilla en papel a la plantilla en la Web (100\%). En la fase 3 se observa un porcentaje de utilización de las TIC del $80,53 \%$. De este total, un 9,29\% corresponde a la profesora y un 90,71\% a los alumnos. La profesora utiliza las TIC como contenido de aprendizaje y, en menor medida, para definir el espacio y procedimiento de realización de la tarea. Los alumnos, por su parte, realizan prioritariamente un uso instrumental de las TIC consistente en copiar en el ordenador un texto previamente elaborado en papel.

Por lo que respecta a la relación entre usos y formas de organización de la actividad conjunta, la Tabla 2 muestra que los distintos tipos de uso se incardinan en formas distintas de organización de la actividad conjunta, mediando de diferentes maneras las relaciones de los participantes entre sí y con el contenido o tarea de aprendizaje. Así, en la fase 1, las TIC median fundamentalmente la relación de los alumnos con el contenido (como se manifiesta en el $83,86 \%$ de uso de las TIC como instrumento cognitivo), y en menor medida las relaciones entre la profesora, los alumnos y el contenido $(8,25 \%$ de uso de las TIC para definir la tarea y 1,08\% como apoyo a la acción docente). Como puede verse, en esta fase las TIC apenas se usan para mediar la comunicación entre los participantes (0,5\%). En la fase 2, las TIC median exclusivamente la relación entre alumnos en torno al contenido o tarea de aprendizaje (100\% de uso instrumental). En la fase 3, la mediación prioritaria es también entre alumnos en torno al contenido o tarea de aprendizaje (76,60\% de uso instrumental y $14,11 \%$ de uso cognitivo), y en menor medida de las relaciones entre la profesora, los alumnos y el contenido o tarea de aprendizaje (7,33\%); de nuevo, en esta fase las TIC se usan muy escasamente para mediar la comunicación entre los participantes $(0,96 \%)$.

Tomados en conjunto, estos resultados muestran el uso específico que se hace de las TIC en el marco de determinadas formas de organización de la actividad conjunta y de las tareas específicas que resuelven. Asimismo, ponen de relieve las mediaciones que introducen en las relaciones entre la profesora, los alumnos y los contenidos de aprendizaje en la secuen- 
cia. Sin embargo, una comprensión global de los usos de las TIC en esta situación de enseñanza y aprendizaje presencial exige, desde nuestro punto de vista, ampliar la mirada y ubicar los usos descritos en el conjunto de formas de organización de la actividad conjunta que profesora y alumnos van construyendo, tanto las que incluyen el uso de las TIC como las que se desarrollan sin su presencia.

Análisis de la actividad conjunta en la secuencia instruccional: Segmentos de actividad conjunta (SAC) con y sin el uso de las TIC

La aplicación del modelo de análisis de la interactividad (Coll, Colomina, Onrubia y Rochera, 1992) a los datos registrados nos ha permitido identificar la estructura global de la actividad conjunta en cada una de las tres fases de desarrollo del proyecto (véase Tabla 3). La panorámica general de esta estructura muestra la combinación de actividades, con y sin el uso de las TIC, a lo largo de la secuencia instruccional. Desde un punto de vista temporal, esta estructura muestra la existencia en cada una de las tres fases de momentos iniciales que se desarrollan sin el uso de las TIC y que se dirigen a orientar el trabajo de los alumnos en relación con distintos aspectos de la tarea, seguidos de momentos en que los participantes utilizan las tecnologías para resolver distintas tareas involucradas en el desarrollo del proyecto.

La fase 1 tiene como finalidad la construcción de un conocimiento inicial compartido entre la profesora y los alumnos sobre el proyecto Edumet y sobre las escuelas participantes, así como la organización de pequeños grupos de trabajo. La fase comienza con una serie de segmentos de actividad conjunta (SAC) que se desarrollan sin el uso de las TIC, relacionados con la presentación del proyecto por parte de la profesora a todo el grupo clase (SAC de presentación del proyecto), la presentación del contenido curricular (SAC de presentación de tarea, SAC de realización de tarea de estado inicial y SAC de aportación de información), y la organización de los grupos de trabajo (SAC de organización y gestión del grupo). Posteriormente puede observarse la aparición de SAC con el uso de TIC. Estos segmentos están relacionados de nuevo con la presentación del proyecto en la Web Edumet (SAC de presentación del proyecto), la presentación de las escuelas y la elaboración de un texto de presentación para ser enviado a las otras escuelas que los alumnos llevan a cabo en grupos (SAC de presentación de tarea y SAC de elaboración de la información en pequeño grupo). 
Tabla 3. Estructura global de la actividad conjunta: Segmentos de actividad conjunta sin TIC y con TIC en las tres fases de la secuencia instruccional

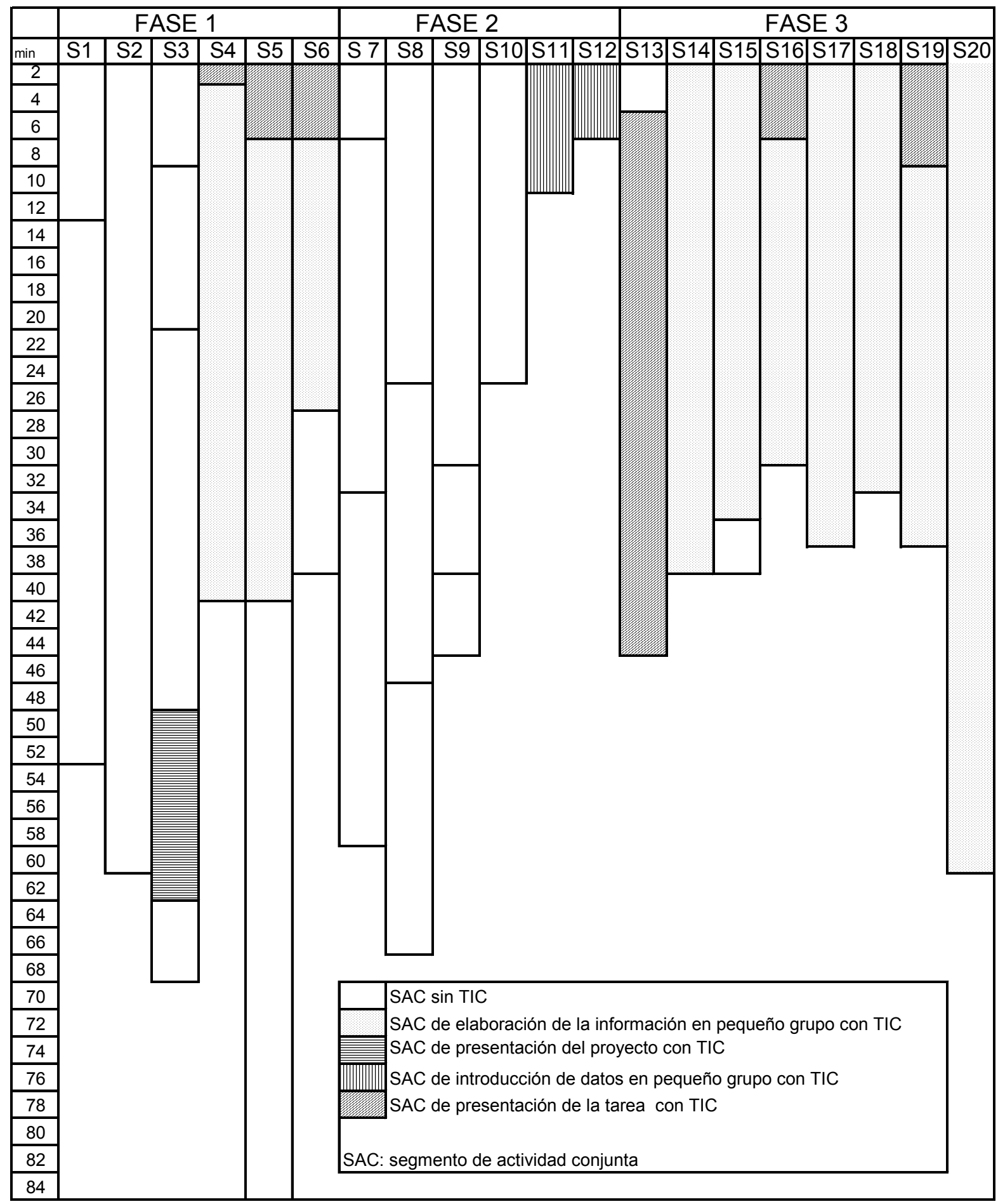

En la fase 2, cuya finalidad es la recogida y almacenamiento de datos meteorológicos, la mayor parte de los segmentos se han desplegado sin el uso de las TIC. Aparecen al inicio segmentos de aportación de información de la profesora a todo el grupo clase -sobre los instrumentos y protocolos en papel para la recogida y lectura de datos meteorológicos- y seg- 
mentos de recogida y almacenamiento de la información -cuando se ejecuta el protocolo en pequeño grupo-. Estos episodios de actividad conjunta ofrecen un contexto simulado para que los alumnos aprendan el procedimiento de recogida y almacenamiento de los datos in situ sobre el clima y el tiempo meteorológico propio del método científico. Al finalizar la fase, y una vez que los alumnos conocen este procedimiento, en pequeño grupo introducen la información en la base de datos en línea y utilizan las TIC de forma contextualizada al simular de nuevo una parte del proceso científico (SAC de introducción de datos en pequeño grupo con TIC).

La fase 3, dirigida a analizar los resultados, elaborar las conclusiones y comunicarlas, se desarrolla casi por completo con uso de TIC, con una organización de los alumnos trabajando por parejas. Tras un momento inicial en el que la profesora ayuda a organizar los grupos de trabajo sin el uso de TIC (SAC de organización y gestión del grupo), aparecen diferentes segmentos de actividad conjunta con uso de TIC dirigidos a la presentación del guión del proyecto de investigación a todo el grupo clase (SAC de presentación de la tarea) y a su elaboración en pequeño grupo (SAC de elaboración de información en pequeño grupo). Para interpretar estos datos, hay que tener en cuenta que se trata de una fase cuyo objetivo consiste en elaborar un informe de investigación a partir de los datos meteorológicos que contiene la base de datos previamente construida, utilizando las TIC cuando los alumnos lo necesitan en función de los requerimientos de la tarea: por ejemplo, para construir el texto escrito, realizar las tablas o elaborar la portada.

Globalmente, los usos de las TIC identificados en la secuencia aparecen vinculados a las necesidades y demandas del proceso de aprendizaje en un entorno de simulación de las fases de la metodología científica que plantea el proyecto Edumet. Se trata, por tanto, de usos contextualizados de las TIC en tareas caracterizadas por su autenticidad, al acercar a los alumnos al uso real de la medición y al análisis de datos meteorológicos en una situación similar a la de una investigación científica. Esos usos, además, parecen mediar de diferentes maneras las formas de organización de la actividad conjunta que van construyendo los participantes, y evolucionan a medida que se desarrolla el proyecto aportando nuevas posibilidades de aprendizaje a los alumnos: en concreto, aparecen usos de las TIC para enseñar TIC, para enseñar con TIC distintos contenidos de área vinculados a la conducción de un estudio sobre el clima y la meteorología, para aprender estos contenidos con TIC en el seno de pequeños grupos de trabajo, o para comunicarse con TIC con otras escuelas. 


\section{Discusión y Conclusiones}

A partir de los resultados descritos es posible identificar algunas claves para comprender los usos educativos que la profesora y los alumnos hacen de las TIC en la secuencia estudiada, el rol mediador de estas tecnologías y de la profesora en el proceso de enseñanza y aprendizaje, y las posibilidades que encierran las tecnologías cuando se usan de manera contextualizada para mejorar, y eventualmente transformar, las prácticas educativas.

Los resultados de nuestro estudio muestran que los usos de las TIC identificados se insertan en el marco de formas diferentes de organización de la actividad conjunta que van construyendo y desarrollando los participantes, creando así posibilidades y restricciones específicas para su actuación, y en particular para los modos en que la profesora puede ayudar a sus alumnos mediando, orientando y guiando los procesos de construcción de conocimiento. Estos usos apuntan a las diferentes direcciones en las que puede desplegarse la potencialidad mediadora de las TIC en los procesos instruccionales. En primer lugar, se usan las TIC para mediar las relaciones entre los alumnos y los contenidos de aprendizaje: es el caso del uso que hacen los alumnos de las TIC como herramientas cognitivas o como instrumentos tecnológicos. En segundo lugar, se usan las TIC para mediar la relación entre profesor, alumnos y contenido: es el caso del uso de las TIC para definir el espacio y procedimiento de realización de la tarea y para la presentación de contenidos realizada por la profesora. En tercer lugar, se usan las TIC como objeto de aprendizaje, como cuando la profesora explica diferentes contenidos tecnológicos: guardar documentos, elaborar gráficas, elaborar dibujos, etc. En efecto, las características tecnológicas de las TIC intervienen en las características de la tarea a desarrollar y, por lo tanto, es importante que los alumnos dominen tecnológicamente el instrumento que va a permitirles realizar correctamente la tarea, razón por la cual la profesora no prescinde de la enseñanza y aprendizaje de los aspectos que considera relevantes para tal desempeño. Finalmente, y aunque en menor caso en esta investigación, otros usos de las TIC identificados remiten a su potencialidad para mediar las interacciones e intercambios comunicativos entre los participantes, como es el caso de su uso para compartir información con otros participantes de diferentes escuelas.

En lo que concierne a la potencialidad de las TIC para mejorar y transformar las prácticas educativas, nuestros resultados confirman resultados previos obtenidos en estudios recientes (por ejemplo, Sigalés, Mominó y Meneses, 2009), al poner de relieve que la incorporación de las tecnologías por sí misma no garantiza la innovación y que no todos los usos 
comportan una mediación y un cambio en profundidad de las interacciones que profesora y alumnos establecen entre sí y con los contenidos que son objeto de enseñanza y aprendizaje. De hecho, una amplia mayoría de los usos reales de las TIC identificados en la secuencia didáctica analizada difícilmente pueden calificarse como transformadores en sentido estricto, si entendemos como tales aquellos usos que promueven el establecimiento de formas de organización de la actividad conjunta novedosas que no serían posibles al margen de los recursos tecnológicos presentes en la situación, como es por ejemplo el caso de los usos de las TIC para la comunicación asíncrona a través de la Web Edumet. Los resultados de nuestro estudio corroboran asimismo los de otros trabajos previos (Barquin, 2007; Zhao y Frank, 2003) respecto a la infrautilización de las potencialidades de las TIC para trasformar la actividad conjunta de los participantes en actividades realmente instruccionales: a pesar de las potencialidades del diseño tecnopedagógico de la Web Edumet para configurar un entorno de aprendizaje colaborativo en línea, el uso real que profesora y alumnos realizan de este entorno es realmente escaso.

La incidencia transformadora e innovadora de las tecnologías en la situación estudiada se vincula más bien al uso contextualizado y auténtico de las TIC en cada fase y momento del desarrollo del proyecto Edumet. Un elemento que refuerza esta interpretación se desprende de la entrevista a la profesora en la que indica que el proyecto tiene por objetivo que los alumnos aprendan fundamentalmente los criterios de uso de las herramientas tecnológicas en la conducción de una investigación científica:

\footnotetext{
"Proyectos como éste (Edumet) que (implican) tres o cuatro meses... me permiten trabajar algunas herramientas, algunos programas [...] y los alumnos han de tener criterio para utilizar la tecnología, [...] con la investigación Edumet, cuando por ejemplo organizan la tabla de datos han de ver cuáles son las columnas que necesitarían, cuáles son las filas, o ver ... qué tipo de gráfico es más representativo de lo que quieren presentar ... la tecnología ha de responder a las necesidades. En Primaria, además han de aprender la herramienta y aprenderla correctamente, pero con prestaciones bajas. O sea que si utilizamos el Excel, utilizamos lo mínimo, cómo se hace un gráfico, cómo se pone un título... que vayan aprendiendo el criterio de utilización de las herramientas"
}

El uso que finalmente acaba realizándose de las TIC, mediando las formas de organización de la actividad conjunta en una u otra dirección, parece depender de la intencionalidad que preside la secuencia didáctica y de las opciones que fundamentan el diseño instruccional de la misma, y no sólo de las características intrínsecas de las TIC. Entre estas opciones cabe 
resaltar la intencionalidad de enfrentar a los alumnos a perspectivas científicas aceptadas en la actualidad y la de utilizar el trabajo entre iguales cómo una forma adecuada de aprender ciencia, dos opciones consideradas fundamentales para la construcción de la comprensión en ciencias naturales en primaria (Thurston, Grant y Topping, 2006; Thurston et al., 2007). Las opiniones expresadas por la profesora en la entrevista final apoyan la interpretación de la importancia de las decisiones del diseño instruccional para la enseñanza y el aprendizaje de la ciencia:

"La tecnología en sí misma no modifica nada ... puedo tener aquí los ordenadores y trabajar de una forma tan clásica y magistral como puedo hacerlo en la clase ... puedes trabajar cooperativamente y no utilizar las TIC ... lo mismo que les hago discutir delante del ordenador lo podian haber discutido delante de una cartulina ... si planteo una cuestión que han de resolver en grupo, el planteamiento es exactamente igual..."

En lo que concierne al papel mediador de la profesora, los resultados sugieren dos grandes ámbitos en los que la intervención docente es decisiva para hacer realidad la potencialidad de las TIC para transformar y mejorar las prácticas educativas. Por una parte, en el ámbito de la planificación y la elaboración del diseño tecnopedagógico (Coll, Mauri y Onrubia, 2008) de la secuencia instruccional, la profesora toma decisiones sobre las actividades más adecuadas para la conducción del proyecto Edumet, la metodología a utilizar, las formas previstas de organización de la actividad conjunta, y muy especialmente sobre cómo se van a utilizar las TIC para lograr las finalidades del proyecto: elaborar textos, construir una base de datos, explicar contenidos y tareas, comunicarse con otras escuelas, etc. Por otra parte, en el ámbito del desarrollo instruccional o pedagógico, la profesora proporciona diversos apoyos y ayudas -con TIC y sin TIC- al proceso de aprendizaje de los alumnos en función de las necesidades que se van originando en cada momento. Esta observación nos acerca por lo demás a la recomendación formulada por Hermans, Tondeur, Van Braak y Valcke (2008) en el sentido de que es necesario que el profesorado desarrolle múltiples competencias vinculadas al uso educativo de las TIC para el logro de los objetivos educativos planteados, a partir de una comprensión constructivista de los procesos de enseñanza y aprendizaje que incluya la mediación del docente.

Para finalizar, y con el fin de seguir profundizando en la potencialidad de las TIC para transformar los entornos educativos y optimizar el aprendizaje de los alumnos, señalar que nuestro trabajo presenta algunas limitaciones al tiempo que ofrece algunas vías para avanzar 
en la comprensión de los factores y dimensiones que intervienen en la mejora de los procesos de aprendizaje con la ayuda de las tecnologías. Por una parte, la naturaleza esencialmente cualitativa y de caso único del trabajo realizado comporta restricciones evidentes para la generalización de las conclusiones que nuevos estudios y aproximaciones de carácter más amplio y extensivo contribuirán sin duda a subsanar. Así, y por referirnos sólo a algunas limitaciones de nuestro trabajo, el estudio de secuencias instruccionales de otras etapas educativas, con otro tipo de contenidos y tareas y en las que se prioricen otros usos de las TIC resulta sin duda necesario para progresar en el estudio de su potencialidad para innovar y mejorar las prácticas educativas.

Por otra parte, si bien centrar el análisis de los usos reales de las TIC en el marco de la actividad conjunta de los participantes constituye una opción nuclear en nuestro planteamiento, hay otros niveles y dimensiones de análisis que es necesario tener en cuenta para alcanzar una visión de conjunto en la comprensión del impacto de las tecnologías en los procesos de aprendizaje. Así, la consideración de un nivel institucional, más macro al analizado en nuestro trabajo, es sin duda necesario para entender algunos de los condicionantes bajo los cuales se construyen los procesos de enseñanza y aprendizaje en el aula. De acuerdo con autores como Fernández y Correa (2008), y como muestran los resultados de nuestro estudio, la flexibilidad del tiempo y del espacio son factores, junto con otras opciones curriculares, que pueden facilitar u obstaculizar de forma importante determinados usos de las TIC. De la misma manera, la toma en consideración de un nivel de análisis más micro al que hemos utilizado en el presente trabajo, centrado en las características de las actuaciones de los participantes y en el contenido de sus intercambios comunicativos, parece fundamental para entender los procesos intrapsicológicos que están implicados en el aprendizaje que lleva a cabo el alumnado en actividades mediadas por las TIC. 


\section{Referencias}

Area, M. (2005). Tecnologías de la información y la comunicación en el sistema escolar. Una revisión de las líneas de investigación. Revista Electrónica de Investigación y Evaluación Educativa, 11(1), 3-25.

Barquin J. (2007). Transiciones en la función docente. La transformación de la práctica educativa por la presencia de los medios electrónicos en la educación. Revista de Educación, 344, 497-509.

Becker, J.H. (2000). Findings from the teaching, learning and computing survey: Is Larry Cuban right? Paper presented at the School Technology Leadership Conference of the Council of Chief State School Officers, Washington, DC.

Blantoon, W.E., Moorman, G., y Trathen, W. (1998). Telecommunications and teacher education: a social constructivist review. Review of Research in Education, 23, 235-275.

Colás, P. y Jiménez, R. (2008). Evaluación del impacto de la formación (online) en TIC en el profesorado. Una perspectiva sociocultural. Revista de Educación, 346, 187-215.

Coll, C. (2001). Constructivismo y educación: la concepción constructivista de la enseñanza y del aprendizaje. En C. Coll, J. Palacios, y A. Marchesi. Desarrollo psicológico y educación. 2. Psicología de la educación escolar, (pp. 157-188). Madrid: Alianza.

Coll, C. (2004). Psicología de la Educación y prácticas educativas mediadas por las tecnologías de la información y la comunicación: una mirada constructivista. Sinéctica, 25, 124.

Coll, C., Colomina, R., Onrubia, J. y Rochera M.J. (1992). Actividad conjunta y habla: una aproximación al estudio de los mecanismos de influencia. Infancia y Aprendizaje, 5960, 189-232.

Coll, C., Onrubia, J. y Mauri, T. (2007). Tecnología y prácticas pedagógicas: las TIC como instrumentos de mediación de la actividad conjunta de profesores y estudiantes. Anuario de Psicología, 38(3), 377-400.

Coll, C., Mauri, T. y Onrubia, J. (2008). La utilización de las tecnologías de la información y la comunicación en la educación: Del diseño tecno-pedagógico a las prácticas de uso. En C. Coll y C. Monereo (Eds.). Psicología de la educación virtual. Enseñar y aprender con las tecnologías de la información y la comunicación (pp. 74-103). Barcelona: Ediciones Morata.

Coll, C., Onrubia, J. y Mauri, T. (2008). Ayudar a aprender en contextos educativos. El ejercicio de la influencia educativa y el análisis de la enseñanza. Revista de Educación, 
346, 33-70.

Coll, C., Rochera, M. J. y Onrubia, J. (2009). De la evaluación continuada hacia la autorregulación del aprendizaje. Algunos criterios y propuestas en la enseñanza superior. En M. Castelló (Coord.). Tendencias actuales de la investigación sobre evaluación auténtica en secundaria y universidad (pp.117-143). Barcelona: Edebé.

Colomina, R., Onrubia, J. y Rochera, M.J. (2001). Interactividad, mecanismos de influencia educativa y construcción del conocimiento en el aula. En C. Coll, J. Palacios, y A. Marchesi (Comps.). Desarrollo psicológico y educación 2. Psicología de la educación (pp. 437-458). Madrid: Alianza.

Darling-Hammond, L., Ancess, J. y Falk, B. (1995). Authentic assessment in action: Studies of schools and students at work. National Center for restructuring education, schools, and teaching, Teachers College, Columbia University: New York.

Díaz Barriga, F. (2005). Enseñanza situada: vínculo entre la escuela y la vida. México: McGraw-Hill.

Fernández, E. y Correa, J.M. (2008). Integración de las TIC en proyectos colaborativos mediante apadrinamientos digitales. Revista Latinoamericana de Tecnología Educativa, 7 (2), 57-67. [http://campusvirtual.unex.es/cala/editio/]

Hermans R., Tondeur, J., Van Braak, J., y Valcke, M. (2008). The impact of primary school teachers' educational beliefs on the classroom use of computers. Computers y Education, 51(4), 1499-1509.

Instituto Nacional De Evaluación y Asesoramiento Educativo, Neturity y Fundación Germán Sánchez Ruipérez (2007). Las tecnologías de la información y de la comunicación en la educación. Informe sobre la implantación y el uso de las TIC en los centros docentes de educación primaria y secundaria (curso 2005-2006). Madrid. Consultado (30.03.2008) en: http://www.oei.es/tic/TICCD.pdf

Marchesi, A. y Díaz, T. (2009). Desafíos de las TIC. El cambio educativo en Iberoamérica. TELOS. Cuadernos de Comunicación e Innovación, Enero-marzo, 78. Consultado (13.02.2009) en:

http://www.campusred.net/telos/articulocuaderno.asp?idarticulo $=6 \mathrm{yrev}=78 \mathrm{\# n} 6$

Muraro, S. (2005). Una introducción a la informática en la escuela. Buenos Aires. Fondo de Cultura Económica de Argentina.

Sigalés, C., Mominó, J.M. y Meneses, J. (2009). TIC e innovación en la educación escolar española. Estado y perspectivas. TELOS. Cuadernos de Comunicación e Innovación, Enero-marzo, 2009, 78. Consultado (13.02.2009) en: 
http://www.campusred.net/telos/articulocuaderno.asp?idarticulo $=4 \mathrm{yrev}=78$

Schrire, S. (2006). Knowledge building as asynchronous discussion groups: Going beyond quantitative analysis. Computers y Education 46, 49-70.

Stake, R.E. (1994). Case Studies. En N.K. Denzin y Y.S. Lincoln (Eds.), Handbook of Qualitative Research (pp.236-247). Thousand Oaks (Cal.): Sage.

Tondeur, J., Van Braak, J. y Valcke, M. (2007). Towards a typology of computer use in primary education. Journal of Computer Assisted Learning, 23, 197-206.

Thurston, A., Grant, G. y Topping, K. J. (2006). Constructing Understanding in Primary Sciences. Electronic Journal of Research in Educational Psychology, 8(4), 1-34.

Thurston, A., Van de Keere, K., Topping, K., Kosack, W., Gatt, S., Marchal, J., Mestdagh, N., Schmeinck, D., Sidor, W. y Donnert, K. (2007). Peer learning in primary school science: Theoretical perspectives and implications for classroom practice. Electronic Journal of Research in Educational Psychology, 13, 5(3), 477-496.

Twining, P. (2002). Conceptualising computer use in education: introducing the Computer Practice Framework (CPF). British Educational Research Journal, 28(1), 95-110.

Yin, R.K. (2002). Case Study Methods. En J.L. Green, G. Camilli y P.B. Elmore (Eds.), Handbook of Complementary Methods in Education Research (pp. 111-122). Mahwah, NJ: L.Erlbaum.

Zhao, Y. y Frank, K.A. (2003). Factors Affecting Technology Uses in Schools: An Ecological Perspectives. American Educational Research Journal, 40(4), 807-840. 